\section{From traditional molecular biology to network oncology}
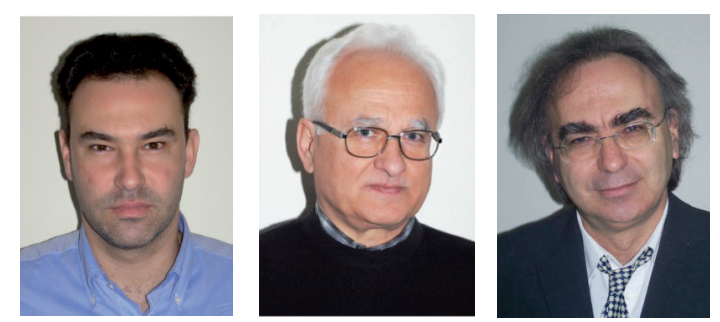

Dimosthenis E Ziogas', Christos Katsios' \& Dimitrios H Roukos ${ }^{\dagger 1,2}$

'Department of Surgery, loannina University School of Medicine, loannina, TK 451 10, Greece

2Personalized Cancer Networks Medicine, Biobank, loannina University, loannina, TK 451 10, Greece

†Author for correspondence: Tel.: +30 2651007423 = Fax: +30 2651007094 = droukos@uoi.gr

Over the past 30 years, molecular biology research has characterized the individual components of a system. In cancer drug development, over the last decade, the emphasis shifted from cytotoxic, nonspecific chemotherapies to molecular tumor-targeted drugs. However, the principle remains the same: targeting a single mutated gene of a biological system - a signaling pathways network - to inhibit signal transduction along a signaling pathway that is involved in cancer development and progression. Nevertheless, despite isolated clinical successes, translation of active drugs into clinical practice remains slow, yielding modest patient benefits. In this article, we discuss the perspectives and limitations of traditional molecular oncology and the expectations and challenges of emerging network-based molecular research.

\footnotetext{
${ }^{\mathbf{6}}$ The eribulin FDA approval is more than just a triumph for patients in desperate need of treatment and represents a hard-won victory for the total synthesis of natural products."
}

Cancer is a major health problem worldwide. Overall, an estimated 12.7 million new cancer cases and 7.6 million cancer deaths occurred in 2008 [1]. The most commonly diagnosed cancers worldwide are lung (1.61 million; $12.7 \%)$, breast $(1.38$ million; $10.9 \%)$ and colorectal cancers (1.23 million; 9.7\%). In the USA, although progress has been made in reducing incidence and mortality rates, cancer still accounts for more deaths than heart disease in persons younger than 85 years [2]. The WHO has estimated that if current trends continue, the global cancer burden will increase by $50 \%$, from 10 million new cases per year in 2000 to 15 million by 2020 [3]. These data suggest that progress in cancer management has been slow and cure rates, particularly in advanced stages II and III, are alarmingly low. Whether more focus and funding on systems biology and network oncology could lead to higher cure rates more quickly than the conventional oncology is addressed in the following section.

\section{Chemotherapy}

Over the last six decades, cytotoxic drugs have been used in the systemic treatment of cancer. Most commonly, chemotherapy acts by killing cells that divide rapidly - one of the main properties of most cancer cells. However, this means that it also harms normal cells, resulting in substantial side effects. At present, standardization of drug schedules and doses have increased response rates and reduced toxicity. For example, adding a taxane to anthracycline-based adjuvant therapy of breast cancer led to an absolute overall survival (OS) benefit of over $30 \%$ [4].

Eribulin - a new nontaxane deep-sea sponge complex synthetic agent - was approved by the US FDA in November 2010 for heavily pretreated patients with metastatic breast cancer. In a Phase III randomized study, the Eisai Metastatic Breast Cancer Study Assessing Physician's Choice Versus Eribulin (EMBRACE) demonstrated a significant OS benefit of 2.5 months in refractory disease to previously treated patients with regimens including anthracycline and taxane [5]. The eribulin FDA approval is more than just a triumph for patients in desperate need of treatment and represents a hard-won victory for the total synthesis of natural products. Therefore, with an absolute net gain in OS of modern chemotherapeutic regimens of approximately 35-40\%, chemotherapy still remains the backbone of cancer treatment, despite its toxicity.

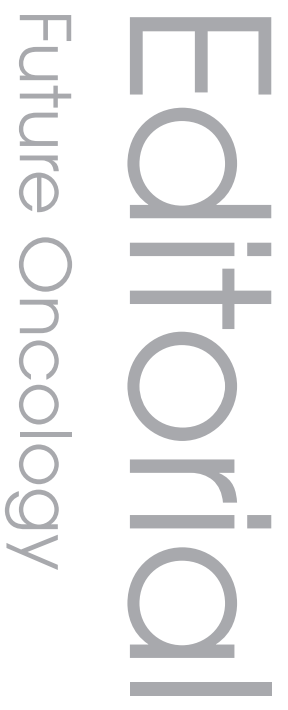

\section{Keywords}

- biological systems

- interactions = molecular

- networks = oncology

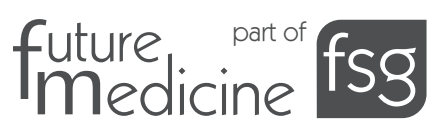




\section{Tumor-targeted agents: moving back} to chemotherapy?

A decade ago, when the first targeted drugs were approved by the FDA for the treatment of patients with advanced cancer, everyone talked about a revolution in translational molecular research. However, 10 years later, Phase III trials testing the promising results by using targeted agents from Phase II trials provide disappointing data. The two main groups of targeted drugs inhibit key signaling pathways, which are deregulated in cancer. The monoclonal antibodies (mAbs) cetuximab and panitumumab block the dimerization of ligand binding to the extracellular receptor domains of the EGF receptor (EGFR) - HER1 and HER2, respectively. The second drug category includes small-molecule tyrosine kinase inhibitors (TKIs), which, when bound to mutated tyrosine kinases, inhibit disturbed signal transduction to the nucleus. Imatinib, gefitinib and erlotinib are the most extensively examinated TKIs. Given that the EGFR regulates hallmark cellular processes, such as cell proliferation, differentiation, survival, apoptosis and angiogenesis, which have been deregulated in cancer, these antiEGFR agents are believed to restore the disturbed biological processes in cancer patients [6].

\section{Isolated clinical success \& resistance}

Targeted therapy has changed multidisciplinary systemic treatment of cancer by adding a targeted drug to standard chemotherapy. Although the advent of a targeted drugs field has revolutionized molecular oncology research and systemic treatment, overall, there has been little improvement in cure rates. From a rapidly growing list of targeted agents approved by the FDA for numerous cancer types, only two drugs have demonstrated a true OS benefit in both the adjuvant and metastatic setting. Imatinib has been shown to have remarkable clinical activity in patients with chronic myeloid leukemia (CML) and malignant gastrointestinal stroma tumors (GISTs), leading to its approval for treatment of these diseases [7,8]. The second is trastuzumab, a $\mathrm{mAb}$ against the HER 2 signaling pathway. This anti-HER 2 drug, used in chemotherapy in the adjuvant treatment of breast cancer and in the metastatic setting for both breast cancer and gastric cancer, has demonstrated significant activity response and an OS benefit by modest adverse effects profile [6,9].

\section{Current disappointments}

Even for these two drugs, primary and acquired resistance pose a substantial problem. Primary and secondary mutations, as well as multiple other molecular mechanisms, have been described to explain resistance to these drugs. Despite initial enthusiasm, more recent Phase III studies investigating other targeted drugs provide disappointing results. The following paragraphs describe more recent modest activity or negative results demonstrated in Phase III studies.

\section{"Even in EGFR-mutant tumors, although TKIs can double survival time compared with wild-type patients, all these patients will unfortunately develop acquired resistance and die from the disease."}

Cetuximab and panitumumab are more recent anti-EGFR (HER1) mAbs. Although there was initial enthusiasm for success in the treatment of colorectal cancer, subsequent more appropriate Phase III trials have limited its efficacy only to genotyping-based selection of patients with wild-type KRAS metastatic colorectal cancer (mCRC) [10]. However, a significant problem of studies testing mAbs or TKIs is that most of these trials have used progression-free survival (PFS) as a primary end point, which today is strongly controversial regarding its objectivity. Mounting evidence now suggests that OS should be used as a primary end point. Indeed, more recent Phase III studies, using OS as primary end point, have provided negative results. Such Phase III studies presented at the 2010 American Society of Clinical Oncology (ASCO) meeting suggest serious concerns. No OS benefit was demonstrated in the adjuvant or metastatic setting, either in the whole group or wildtype $K R A S$ selected subgroup of patients with colorectal cancer treated by adding cetuximab to chemotherapy [11,12].

Erlotinib and gefitinib - the first generation of small-molecule TKIs - have shown an activity and treatment response of less than 15\% among patients with non-small-cell lung cancer (NSCLC). This molecular research-based identification of a subset of patients with EGFR mutations represents a significant advance. Yet, for the remaining $85 \%$ of patients, no targeted agent has been developed to overcome primary resistance. Even in EGFR-mutant tumors, although TKIs can double survival time compared with wild-type patients, all these patients will unfortunately develop acquired resistance and die from the disease [13].

Bevacizumab has become a popular antiVEGF drug that is widely used in clinical practice for many cancer types, although high-quality 
evidence of a true OS benefit is still lacking. Despite this popularity and the approval of bevacizumab (Avastin ${ }^{\circledR}$, Genentech, CA, USA) by the FDA on the basis of more recent studies using OS as the primary end point, the FDA recommends withdrawing bevacizumab as a treatment for metastatic breast cancer [101].

\section{Breaking from the past}

In December 2010, the NIH voted to launch a National Center for Advancing Translational Sciences, focusing on translational medicine and therapeutics (TMAT), the growing field that aims to speed up the translation of therapies from the laboratory to the clinic. NIH director Francis Collins called the decision "momentous" - a "disruptive innovation on an institutional scale" - and he was absolutely right [14]. In a related comment in Nature, Fitzgerald points out that we need to break from the past in order to develop new medicines, and the interdisciplinary NIH TMAT center is an important step forward [14]. But to which research direction, among the numerous strategies from current traditional molecular biology (e.g., stem cells, cancer genomics and functional genetic regulation), should priority be given?

\section{Sequencing cancer genomes}

At the end of the first postgenomic decade, we are now facing a genomic revolution $[15,16]$. The ability of massively parallel genome sequencing technology for the simultaneous analysis of millions of genetic variants across the genome permits the identification of causal mutations underlying common human disorders. This assessment paves the way for understanding of disease pathogenesis, risk prediction, prevention and treatment attracting major interest from biotechnology, the pharmaceutical industry, biomedical sciences and academia.

\footnotetext{
"Strong emerging evidence indicates that cancer and other common disease initiation and progression processes are driven by gene-gene (transcriptome), protein-protein (interactome) and gene-environment interactions rather than a simple accumulation of causal genetic variants..."
}

The capacity of next-generation sequencing (NGS) technology for complete analysis of human or cancer genomes, including both protein-coding and noncoding DNA, can lead to the completion of a mutations catalog for many major diseases, including cancer [17]. The rapidly decreasing costs have led to the completion of approximately 3500 human whole-genomes in 2010 and an estimated 30,000 will be sequenced at the end of 2011 [18]. The recently launched International Cancer Genome Consortium (ICGC) will reveal the repertoire of driver mutations for many cancer types [17]. The ICGC will coordinate systematic large-scale cancer genome studies in more than 25,000 cancer genomes at the genomic, epigenomic and transcriptomic levels. Genome sequencing will be performed in tumors from 50 different cancer types and/or subtypes that are of clinical and societal importance across the globe. Recent systematic studies of cancer genomes reveal that cancer is much more heterogeneous and complicated than we thought.

The unprecedented ability of NGS for discovering most or even the whole set of causal mutations underlying major diseases such as cancer will dramatically improve our knowledge of genomic structural variation underlying the pathogenesis, evolution and therapy response of major diseases. However, how genetic regulation and functional organization of the human genome operates and how deregulation leads to diseases represent the greatest challenges in current and future research [19]. Over the coming years, we can expect an exponential rise in the number of completely sequenced cancer genomes of patients with early or advanced cancer for many cancer types [17]. However, whether this wide range of information and data on the cancer genome alone will lead to novel and effective anticancer therapeutics without understanding functional disturbance in cancer is hard to predict.

\section{Cancer networks \& network molecular oncology}

Despite more recent intensive research efforts, understanding and elucidating complex interactions of biosystems in nature remains elusive. Mounting evidence suggests that biological, cellular and disease outcomes strongly depend on the complex interactions between genes, proteins, biomolecules and signaling pathways. Exciting research is now underway to predict the inference of complex dynamic biological and environmental systems [19,20]. Indeed, such a prediction is crucial in the field of novel biomarkers and drug development. Strong emerging evidence indicates that cancer and other common disease initiation and progression processes 
are driven by gene-gene (transcriptome), protein-protein (interactome) and gene-environment interactions rather than a simple accumulation of causal genetic variants and epigenetic modifications over a cut-off value [21-24].

These latest data emerging from NGS and other modern high-throughput technologies allow us now to measure biomolecule interactions and networks outcomes, and increasingly understand the role of epigenetic events and miRNAs in networks predictions better. Epigenome alterations have been tested with encouraging results in clinical trials and many other clinical studies are underway to assess the potential clinical utility of epigenetic alterations and miRNA expression pattern measurements as prognostic, predictive and therapeutic targets in solid cancer [25,26].

Although the research in understanding the influence of ecological, social and biological system interactions in humans is still in its infancy, the belief that everything is connected [27] can show us how to change our thinking. Current advances in systems biology and mathematics provide hope for a step-by-step guide to understanding nonlinear complex networks $[28,29]$. The time has arrived to focus more on systems biology [25-28] and move from traditional molecular biology simply characterizes the components of a system of interest, to more

sophisticated studies of the complex dynamic interactions of these components (genes, proteins and biomolecules) [27].

\section{Conclusion}

Although exciting discoveries have been achieved by traditional molecular oncology, few of these advances have been translated with clinical success in improving health. Unfortunately, US and WHO cancer statistics confirm that progress in improving cancer patients' outcomes is slow.

The advent of the latest high-throughput technologies permits measurements of complex biosystems interactions and we are now starting to move from ideas and concepts of the significance of networks research to scientific evidence. It is now the time to break the past and move forward with network molecular oncology.

\section{Financial \& competing interests disclosure \\ The authors have no relevant affiliations or financial involvement with any organization or entity with a financial interest in or financial conflict with the sub- ject matter or materials discussed in the manuscript. This includes employment, consultancies, honoraria, stock ownership or options, expert testimony, grants or patents received or pending, or royalties. \\ No writing assistance was utilized in the production of this manuscript.}

\section{Bibliography}

1. Ferlay J, Shin HR, Bray F, Forman D Mathers C, Parkin DM: Estimates of worldwide burden of cancer in 2008: GLOBOCAN 2008. Int. J. Cancer DOI: 10.1002/ijc.25516 (2010) (Epub ahead of print).

2. Jemal A, Siegel R, Xu J, Ward E: Cancer statistics, 2010. CA Cancer J. Clin. 60(5), 277-300 (2010).

3. Lingwood R, Boyle P, Milburn A et al. The challenge of cancer control in Africa. Nat. Rev. Cancer 8, 398-403 (2008).

4. Martin M, Segui MA, Anton A et al.; GEICAM 9805 Investigators: Adjuvant docetaxel for high-risk, node-negative breast cancer. N. Engl. J. Med. 363(23), 2200-2210 (2010).

5. Twelves C, Loesch D, Blum JL et al.: A Phase III study (EMBRACE) of eribulin mesylate versus treatment of physician's choice in patients with locally recurrent or metastatic breast cancer previously treated with an anthracycline and a taxane. J. Clin. Oncol. 28(Suppl. 18), Abstract CRA1004 (2010).
6. Baselga J, Swain SM: Novel anticancer targets: revisiting ERBB2 and discovering ERBB3. Nat. Rev. Cancer 9(7), 463-475 (2009).

7. Waller CF: Imatinib mesylate. Recent results. Cancer Res. 184, 3-20 (2010).

8. An X, Tiwari AK, Sun Y, Ding PR, Ashby CR Jr, Chen ZS: BCR-ABL tyrosine kinase inhibitors in the treatment of Philadelphia chromosome positive chronic myeloid leukemia: a review. Leuk. Res. 34(10), 1255-1268 (2010).

9. Bang YJ, Van Cutsem E, Feyereislova A; ToGA Trial Investigators: Trastuzumab in combination with chemotherapy versus chemotherapy alone for treatment of HER2-positive advanced gastric or gastro-oesophageal junction cancer (ToGA): a Phase 3, open-label, randomised controlled trial. Lancet 376(9742), 687-697 (2010).

10. Van Cutsem E, Köhne CH, Hitre E et al.: Cetuximab and chemotherapy as initial treatment for metastatic colorectal cancer. N. Engl. J. Med. 360 (14), 1408-1417 (2009).

11. Alberts. SR, Sargent DJ, Smyrk TC et al.: Adjuvant mFOLFOX6 with or without cetuximab (Cmab) in KRAS wild-type (WT) patients (pts) with resected stage III colon cancer (CC): results from NCCTG Intergroup Phase III trial N0147. J. Clin. Oncol. 28(Suppl. 18), Abstract CRA3507 (2010).

12. Maughan. TS, Adams R, Smith CG et al.; MRC COIN Trial Investigators: Identification of potentially responsive subsets when cetuximab is added to oxaliplatin-fluoropyrimidine chemotherapy (CT) in first-line advanced colorectal cancer (aCRC): mature results of the MRC COIN trial. J. Clin. Oncol. 28(Suppl. 15), Abstract 3502 (2010).

13. Pao W, Chmielecki J: Rational, biologically based treatment of EGFR-mutant non-smallcell lung cancer. Nat. Rev. Cancer 10(11), 760-774 (2010).

14. Fitzgerald G: Drug development needs a new brand of science. Nature 468(7326), 869 (2010).

15. Collins F: Has the revolution arrived? Nature 464(7289), 674-675 (2010).

16. Venter JC: Multiple personal genomes await. Nature 464 (7289), 676-677 (2010).

17. International Cancer Genome Consortium: International network of cancer genome projects. Nature 464, 993-998 (2010). 
18. Human genome: genomes by the thousand. Nature 467(7319), 1026-1027 (2010).

19. Heard E, Tishkoff S, Todd JA et al.: Ten years of genetics and genomics: what have we achieved and where are we heading? Nat. Rev. Genet. 11(10), 723-733 (2010).

20. Schadt EE: Molecular networks as sensors and drivers of common human diseases. Nature 461(7261), 218-223 (2009).

21. Tamsir A, Tabor JJ, Voigt CA: Robust multicellular computing using genetically encoded NOR gates and chemical 'wires'. Nature DOI: 10.1038/nature09565 (2010) (Epub ahead of print).

22. Morris MK, Saez-Rodriguez J, Sorger PK, Lauffenburger DA: Logic-based models for the analysis of cell signaling networks. Biochemistry 49, 3216-3224 (2010).
23. Bonetta L: Protein-protein interactions: interactome under construction. Nature 468(7325), 851-854 (2010).

24. Breitkreutz A, Choi H, Sharom JR et al:: A global protein kinase and phosphatase interaction network in yeast. Science 328(5981), 1043-1046 (2010).

25. Garzon R, Marcucci G, Croce CM: Targeting microRNAs in cancer: rationale, strategies and challenges. Nat. Rev. Drug. Discov. 9(10), 775-789 (2010).

26. Kelly TK, De Carvalho DD, Jones PA: Epigenetic modifications as therapeutic targets. Nat. Biotechnol. 28(10), 1069-1078 (2010).

27. Beltrao P, Cagney G, Krogan N: Quantitative genetic interactions reveal biological modularity. Cell 141(5), 739-745 (2010).
28. Zheng J, Benschop JJ, Shales M et al.: Epistatic relationships reveal the functional organization of yeast transcription factors. Mol. Syst. Biol. 6, 420 (2010).

29. Wood SN: Statistical inference for noisy nonlinear ecological dynamic systems. Nature 466(7310), 1102-1104 (2010).

\section{Website}

101. BLA 125085 Avastin (bevacizumab) withdraw memo: metastatic breast cancer indication http://google2.fda.gov/search?q=cache:mIqSHElxMEJ:www.fda.gov/ downloads/Drugs/DrugSafety/ PostmarketDrugSafetyInformationfor PatientsandProviders/UCM237171.pdf+beva cizumab+withdrawn \&client $=$ FDAgov $\&$ site $=$ FDAgov\&lr $=$ \&proxystylesheet=FDAgov\&ou tput $=x m l \_n o \_d t d \&$ ie $=$ UTF-

$8 \&$ access $=$ p \&oe $=$ UTF -8 